\title{
Scaling behavior in disordered sandpile automata
}

\author{
B. Tadić, ${ }^{*}$ U. Nowak, ${ }^{\dagger}$ and K.D. Usadel \\ Theoretische Tieftemperaturphysik, Universität Duisburg, Lotharstrasse 1, \\ 4100 Duisburg, Germany \\ R. Ramaswamy \\ School of Physical Sciences, Jawaharlal Nehru University, New Delhi 110067, India \\ S. Padlewski \\ Theoretical Condensed Matter Physics, University of Cambridge, Cavendish Laboratory, \\ Madingley Road, CB3 OHE Cambridge, England
}

(Received 3 December 1991)

\begin{abstract}
We study numerically the scaling behavior of disordered sandpile automata with preferred direction on a two-dimensional square lattice. We consider two types of bulk defects that modify locally the dynamic rule: (i) a random distribution of holes, through which sand grains may leavc the system, and (ii) several models with a random distribution of critical heights. We find that at large time and length scales the self-organized critical behavior, proved exactly in the pure model, is lost for any finite concentration of defects both in the model of random holes and in those models of random critical heights in which the dynamic rule violates the height conservation law. In the case of the random critical height model with the height-conserving dynamics, we find that self-organized criticality holds for the entire range of concentrations of defects, and it belongs to the same universality class as the pure model. In the case of random holes we analyze the scaling properties of the probability distributions $P(T, p, L)$ and $D(s, p, L)$ of avalanches of duration larger than $T$ and size larger than $s$, respectively, at lattices with linear size $L$ and concentration of defect sites $p$. We find that in general the following scaling forms apply: $P(T)=T^{-\alpha} \mathcal{P}(T / x, T / L)$ and $D(s)=s^{-\tau} \mathcal{D}\left(s / m, s / L^{\nu}\right)$, where $x \equiv x(p)$ and $m \equiv m(p)$ are the characteristic duration (length) and the characteristic size (mass) of avalanches for a given concentration of defects. The power-law behavior of the distributions still persists for length scales $T \ll x(p)$ and mass scales $s \ll m(p)$. The characteristic length $x(p)$ and mass $m(p)$ are finite for small concentrations of defects and diverge at $p \rightarrow 0$ according to the power law $x(p) \sim p^{-\mu_{x}}$ and $m(p) \sim p^{-\mu_{m}}$, with the numerically determined values of the exponents close to $\mu_{x}=1$ and $\mu_{m}=1.5$. The finite-size of the lattice may affect the measured probability distributions if for a given concentration of defects the characteristic length $x(p)$ cxceeds the lattice sizc $L$. $A$ finite-size scaling analysis for the mass distribution yields the exponent $\nu=1.5$, while the duration of the avalanches scales linearly with the size. We also determine the exponent $D=1.5$ that connects the mass and the duration of avalanches.
\end{abstract}

PACS number(s): 05.40.+j, 64.60.Fr, 64.60.Ht

\section{INTRODUCTION}

In a recent study of the response in extended dissipative dynamic systems, Bak, Tang, and Wiesenfeld [1] introduced the notion of self-organized criticality (SOC), which is now believed to represent a large class of phenomena with generic scale invariance [2]. Various models of self-organized criticality have been introduced and studied both analytically and numerically [1-10]. Among those, the most intensively studied are sandpile automata, first introduced in Ref. [1], which are believed to describe the universal properties of the self-organized critical state. In a sandpile automaton sand grains are repeatedly added from the outside and the system evolves according to simple dynamic rules, e.g., sand slides from an unstable site $(i, j)$ if the height measured in the number of sand grains at that site exceedes some critical value $h_{c}(i, j)$. The sand is transferred to the neighboring sites which may render them unstable and thus a cascade of sand slidings (an avalanche) may be created. When an avalanche reaches the boundary some sand grains are thrown out of the system. The total mass of the system oscillates around a well-defined average value when the steady state is reached. When hit by avalanches, the height at a given lattice site $h(i, j)$ fluctuates around the mean value $\langle h\rangle$, which defines a flat profile of the sandpile. After long time the stochastic evolution develops a state which is characterized by avalanches of all sizes and durations. The lack of an intrinsic length and time scale shows analogy to the critical state of a second-order phase transition [11]. However, in contrast to usual phase transitions, the self-organized critical state is thought not to depend on the value of any tuning parameters, but is an intrinsic property of the dynamics of the system.

The integrated probability distribution of an avalanche of duration $T$ and size $s, P(T)$ and $D(s)$, respectively, 
are characterized by the power laws $P(T) \sim T^{-\alpha}$ and $D(s) \sim s^{-\tau}$. Unlike the ordinary critical behavior, the number of independent critical exponents needed to fully describe the self-organized critical state is not known (see, however, Refs. [4] and [5]). It has been recognized recently [5-7] that models of self-organized criticality are characterized by the symmetry with respect to translations of height $h(i, j)$, the dynamics which satisfies a conservation law (e.g., conservation of the number of grains under the microscopic dynamic rule, in the case of sandpile automata), and a nonconserving external noise. Varying the microscopic dynamic rule which describes how an avalanche is generated, the symmetry properties, or the type of external noise that triggers the response of the system, may lead to different universality classes of the self-organized criticality, which are defined by the critical exponents and the corresponding scaling functions.

Recently, Manna [8] has found numerically that in twodimensional sandpile automata which obey different dynamic rules, such as the critical height model (CHM), the critical gradient model, and the Laplacian model, the self-organized critical state is characterized by different critical exponents. Kadanoff et al. [9] have determined the exponents and the corresponding scaling functions in one- and two-dimensional sandpile automata with various dynamic rules. Dhar and Ramaswamy [10] have calculated exactly the critical exponents for the critical height model with preferred direction in all dimensions. In two dimensions, for instance, the above-defined exponents are $\alpha=1 / 2$ and $\tau=1 / 3$ for the directed CHM. Hwa and Kardar [5] have introduced a continuum model which describes fluctuations around a steady state in a flowing sandpile. Using some general properties of the dynamic renormalization-group scaling equations in this model, they have shown that the self-organized criticality can be understood in terms of a conservation law in dynamics, and have calculated the critical exponents exactly in all dimensions. Manna, Kiss, and Kertész [12] have studied the model of SOC in which the local conservation law is violated. They found that when the transfer ratio is allowed to fluctuate around some critical value, but is conserved globally (local violation), SOC with mean-field-like exponents is observed, whereas considering the global violation of the conservation law, they found that the system is driven out of criticality. Regarding the necessity of a conservation law see also Ref. [13].

It has been argued recently by Toner [14] that defects which locally break the translational symmetry in the interior bulk of sandpiles are relevant for their surface fluctuations. Using the dynamic renormalization group in the Hwa-Kardar model with quenched defects, he found that in the long-distance and long-time limit the fluctuations around a steady state of the sandpile profile are described by a simple diffusion equation with noise. This means that SOC survives with mean-field-like exponents. The question of randomness in the sandpile automata was addressed by Bak, Tang, and Wiesenfeld in Ref. [3]. They studied the cluster size distribution by removing bonds at random in the isotropic cellular automaton of size $20 \times 20$. They found power-law behavior with the same exponent as for the pure system.

In the present work we use numeric simulations to study the effect of bulk disorder on the self-organized critical state in the critical height model with preferred direction. We consider two types of defects: (i) the random-holes (sinks) model, where holes, through which sand particles may leave the system, are randomly distributed in the underlying lattice, and (ii) the random critical height model, where the toppling condition depends on the local critical height. As it will be described below, in our models both the conservation of heights and the translational symmetry are locally violated in the presence of defects. Our results suggest that in this case the self-organized criticality is lost at large scales and large times. To check up the importance of the violation of the height conservation, we study comparatively a second model with random critical heights, in which the dynamic rule is changed in such a way that the flux of particles at each site is kept constant. We find that for all concentrations of defects this model shows self-organized criticality with the critical exponents of the pure directed model.

In the case of random holes we study the details of the loss of self-organized criticality by varying the concentration of defects. For a small concentration of defects ( $p<p^{*}$, where $p^{*}$ is approximately equal to the critical concentration for directed percolation on the square lattice [15]), the self-similarity which characterizes the critical state persists on scales smaller than the characteristic screening length $x(p)$ or mass $m(p)$. We find that the characteristic length $x(p)$ and the characteristic mass $m(p)$ diverge as a power of the concentration of defects $p$ when $p \rightarrow 0$. We investigate the scaling behavior of the probability distributions $P(T, p, L)$ and $D(s, p, L)$ for small concentrations $p$ and various system sizes $L$, and we determine the corresponding scaling exponents.

The organization of the paper is as follows. In Sec. II we describe the simulations and present the results in the case of the random-holes model. The scaling analysis of the data is given in Sec. III. In Sec. IV we present results of simulations in the random critical height model. A short summary and a discussion of the results are given in Sec. V.

\section{RANDOM-HOLES MODEL: SIMULATIONS AND RESULTS}

We consider the critical height model on a square lattice with an integer variable $h(i, j)$ associated with each lattice site. The dynamics of the model is discrete, diffusive, and supplemented by the preferred direction. It is defined by the microscopic dynamic rule that if at any site $h(i, j)$ exceeds the critical value $h_{c}(i, j)$,

$$
h(i, j) \geq h_{c}(i, j)
$$

then the local height $h(i, j)$ decreases. In the present model we assume that all lattice sites have the same critical height $h_{c}(i, j)=2$. Then the local height decreases by 2 and the heights at its two forward neighboring sites 
$j_{ \pm}=j \pm\left[1 \pm(-1)^{i}\right] / 2$ increase by 1 :

$h(i, j) \rightarrow h(i, j)-h_{c}(i, j) \quad h\left(i+1, j_{ \pm}\right) \rightarrow h\left(i+1, j_{ \pm}\right)+1$.

The self-organized critical behavior in the directed critical height model has been studied analytically [10]. It has been shown that the existence of the preferred direction leads to a new universality class, compared to the undirected model.

In this section we study the directed critical height model on a two-dimensional square lattice in which a fraction of sites $p$ are considered as holes. The sand grains may leave the system through these holes during the dynamic evolution. The distribution of defect sites is random and can be considered as either "annealed" or "quenched." In the case of "annealed" disorder we fix the probability $p$ that a defect may occur at each lattice site during one event (development of an avalanche following one added particle at the top line). The distribution of defects is thus generated randomly for each event, and is not memorized for the next event, when a new distribution with the same concentration of defects $p$ is generated. "Quenched" defects are implemented such that a distribution of defect sites is generated for each concentration $p$ and held fixed during all events. The process is repeated for an ensemble of distributions of defects and the measured properties are eventually averaged over all distributions of defects.

We start with random initial conditions, i.e., a halffilled lattice. Then a particle is added at one randomly chosen site at the first row. If at that site the condition (2.1) is fulfilled, then two particles slide to the two nearest neighbors at the next row, where the toppling condition may be fulfilled again, and so on. Thus one added particle at the first row may cause rearrangements at all scales (an avalanche is created). We follow an avalanche until the system regains its stability, and then the next particle is added at the top. Sand grains are allowed to leave the system either when an avalanche reaches the lower boundary or through the holes. We employ periodic boundary conditions in the perpendicular direction. The avalanche is characterized by the duration $T$-number of rows on which slidings occur, and the mass $s$-total number of sites that undergo slidings following one added particle at the top. In the absence of defects the probability distributions $P(T)$ of an avalanche longer or equal to $T$ and the probability distribution $D(s)$ of avalanches having a mass which exceeds $s$ falls off at large $T$ and $s$ as $P(T) \sim T^{-1 / 2}$ and $D(s) \sim s^{-1 / 3}$, respectively [10].

A typical number of events in our simulations is $5 \times 10^{6}$, where the first $1 \times 10^{6}$ steps are left for the system to reach the steady state. We used lattices of linear size $L$ that range from 20 to 1000 .

The results for the probability distributions $P(T)$ and $D(s)$ are given in Figs. 1(a) and 1(b), respectively, for different concentrations of defects $p$. The upper curves correspond to the lattice without defects $(p=0.0)$, and the following curves from the top to bottom are for $p=0.0075$, $0.01,0.025,0.05,0.1,0.2$, and 0.295 . The lattice sizes used in these simulations are $1000 \times 1000$ for the case of small concentrations $p \leq 0.05$, and $100 \times 100$ for the higher concentrations of defects. The data are from the model with annealed disorder. In the absence of defects $(p=0)$ we observe a power-law behavior of $P(T)$ and $D(s)$ [slope in the upper curve in Fig. 1(a), and Fig. 1(b)], with the exponents $\alpha=0.4950 \pm 0.0001$ and $\tau=0.3319 \pm 0.0004$. The exactly known distributions [10] follow power laws with exponents $1 / 2$ and $1 / 3$, respectively, in the limit of infinite durations. The systematic deviations in our numeric values compared to these limiting laws are due to the finite durations in the
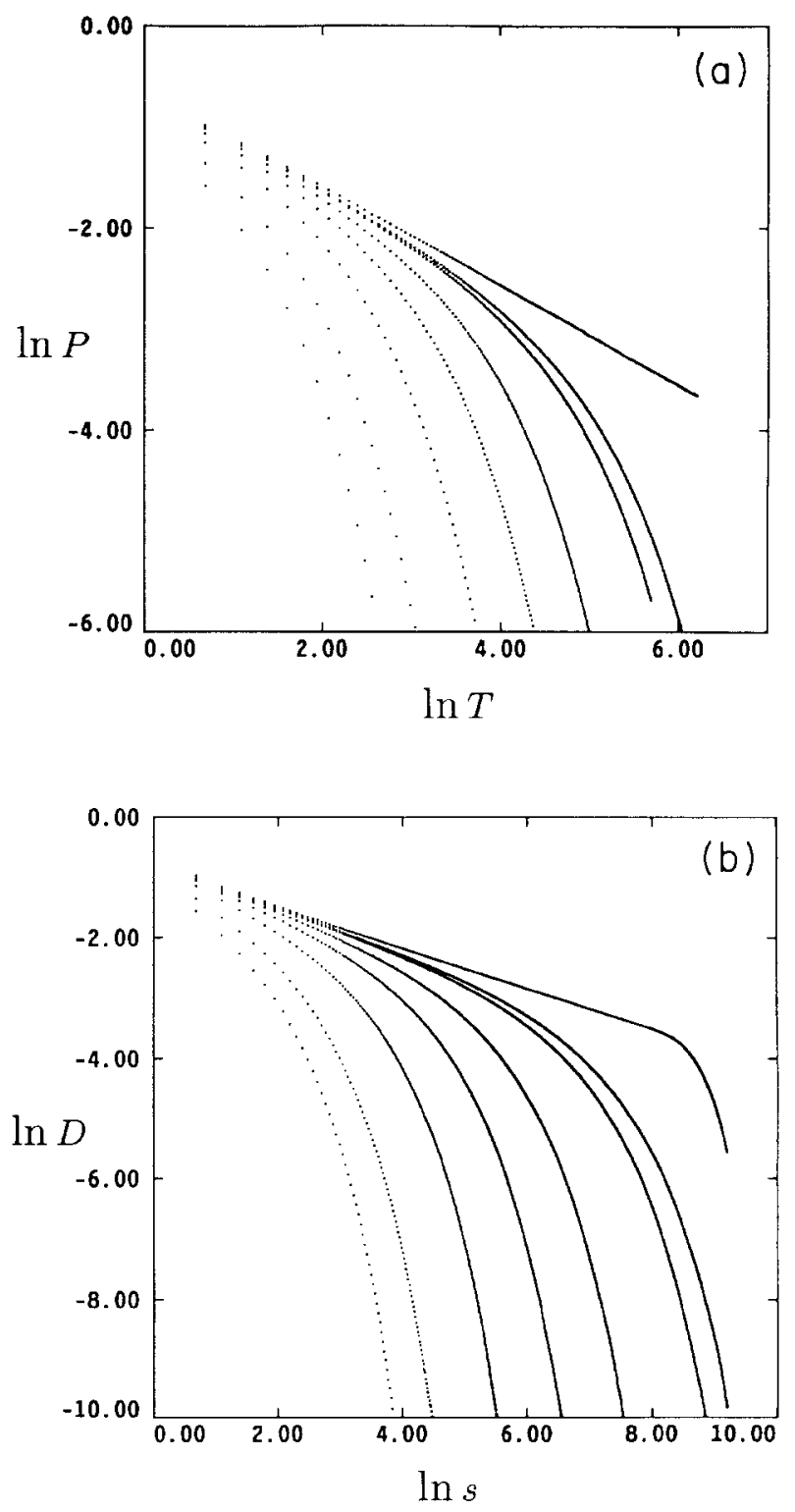

FIG. 1. (a) Double-logarithmic plot of the probability distribution $P$ normalized to the total number of events against the duration of the avalanches $T$ for different values of the concentrations of defects (from top to bottom) $p=0.0,0.0075$, $0.025,0.05,0.1,0.2$, and 0.295 . (b) Same as (a) but for the distribution $D$ against the size of the avalanches. 
simulation. As the results in Fig. 1 suggest, the powerlaw behavior is last at large $T$ and large $s$ for any finite value of the concentration of defects. For small concentrations, however, there are finite regions of parameters $T$ and $s$ where the power-law behavior still holds. By fitting the curves in Fig. 1(a) to the general expression $P(T, p)=A T^{-1 / 2} \exp (-T / x)$, we determine the length $x(p)$, which screens the long-range power-law behavior of the distribution. In Fig. 2 (lower curve) the screening length $x(p)$ is plotted against concentration of defects in a double-logarithmic scale. The upper curve in Fig. 2 is the result of a similar fit of the mass distribution from Fig. 1(b), namely, $D(s, p)=B s^{-1 / 3} \exp (s / m)$. In both cases different points correspond to different curves in Figs. 1(a) and 1(b). As the results in Fig. 2 suggest, the characteristic length and the characteristic size of the avalanches in the presence of defects vary as power of the concentration of defects

$$
x(p) \sim p^{-\mu_{x}}, \quad m(p) \sim p^{-\mu_{m}} .
$$

As the best fit we find $\mu_{x}=1.01 \pm 0.02$ and $\mu_{m}=$ $1.52 \pm 0.01$. Our numeric results suggest that Eqs. (2.3) are valid up to some critical concentration $p^{*}$, which is close to the critical value for directed percolation on the square lattice $p_{c}=0.295$ [15], where the largest possible avalanche becomes finite. It should be noted here that our simulations with quenched defects lead to the same values of the exponents defined in Eq. (2.3), apart from a numeric error.

In Fig. 3(a) we present the length (duration) of avalanches averaged over the total number of events $\langle T\rangle$ versus lattice size $L$ for the pure case $(p=0.0)$ and for two different concentrations of random defects $p=0.025$

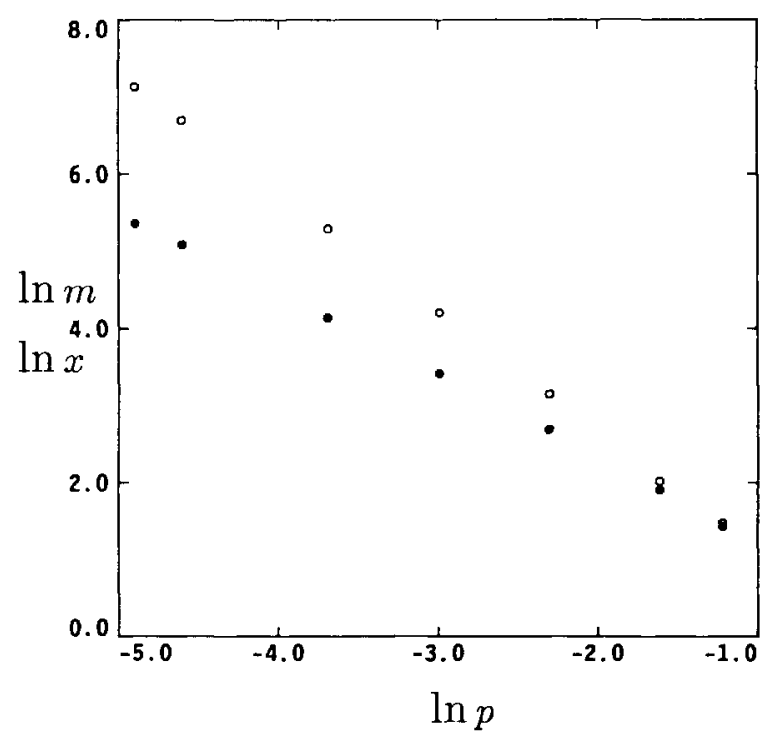

FIG. 2. Double-logarithmic plot of the characteristic length $x$ (full circles) and characteristic mass $m$ (open circles) against the concentration of defects $p$. The values of $p$ correspond to Fig. 1.

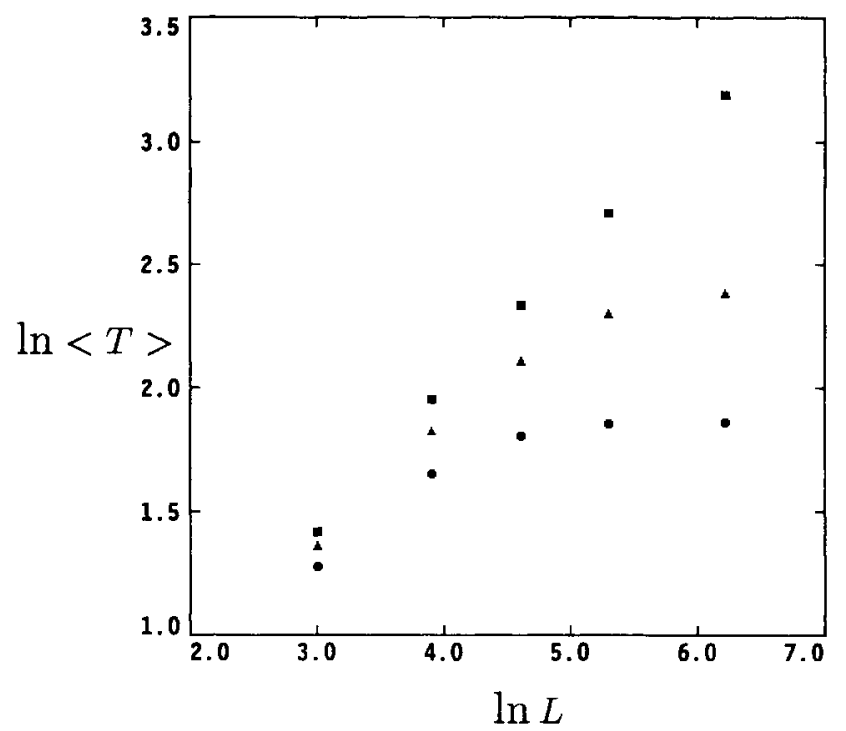

FIG. 3. Double-logarithmic plot of the duration of avalanches averaged over the total number of events $\langle T\rangle$, plotted against the lattice size $L$ for the perfect lattice $p=0.0$ (squares), and two different concentrations of defects $p=0.025$ (triangles) and $p=0.05$ (circles).

and 0.05 . In the pure case the average length scales with the system size as $\langle T\rangle \propto L^{0.529 \pm 0.006}$ (upper curve in Fig. 3). We find that the average size of the avalanche (not shown) scales as $\langle s\rangle \propto L^{1.00002 \pm 0.00001}$. Even though there a systematic deviation from a power law for small $L$ these results are in rough agreement with the values $1 / 2$ and 1, respectively, which follow from the exactly known behavior of the distributions $P(T)$ and $D(s)$ [10].

For finite disorder, however, this scaling is valid only for small values of $L$. When the lattice size exceeds the screening length for a given concentration of defects, $L \gg x(p)$, the average length of the avalanche levels up and the scaling fails. The average size of the avalanche $\langle s\rangle$ shows similar behavior, except that the length $L$ should be compared with $[m(p)]^{1 / D}$, where $D$ is the dimensionality of the typical cluster (see below). In the following section we will investigate the scaling behavior of $P(T)$ and $D(s)$ from a more formal point of view.

\section{SCALING ANALYSIS}

The appearance of a characteristic length $x(p)$ and a corresponding characteristic mass $m(p)$ is the way that the system's response to the external perturbation accommodates to the presence of defects. For lengths smaller than the characteristic length for a given concentration of defects $T \ll x(p)$ the response is still "critical," meaning that the probability distributions fall off as a power of $T$ and $s$. The characteristic values $x(p)$ and $m(p)$ vary with the concentration of defect sites $p$ (cf. Fig. 2), and if for a given concentration the screening length exceeds the system size, e.g., $x(p) \geq L$, then the 
finite size of the lattice may affect the measured probability distribution. In principle, if we scale the length by an arbitrary scaling factor $l>1$, we expect that the probability distributions $P(T, p, L)$ and $D(s, p, L)$ will have the following scaling properties:

$$
P(T, p, L)=l^{\lambda} P\left(l^{\lambda_{T}} T, l^{\lambda_{p}} p, l^{-1} L\right)
$$

and

$$
D(s, p, L)=l^{\lambda^{\prime}} D\left(l^{\lambda_{s}} s, l^{\lambda_{p}} p, l^{-1} L\right),
$$

where $\lambda_{T}, \lambda_{p}$, and $\lambda_{s}$ are corresponding scaling exponents
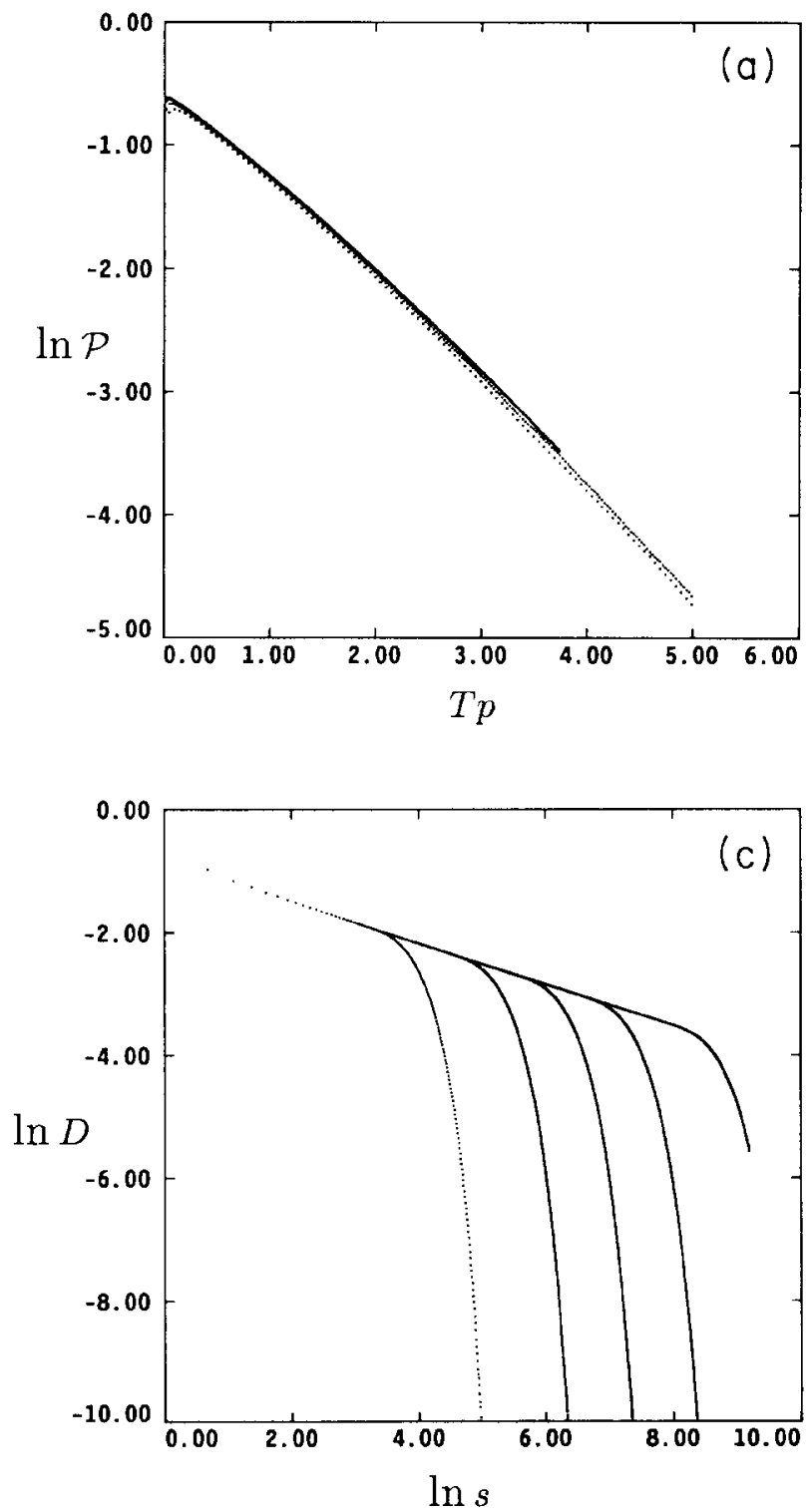

for the duration $T$, concentration of defects $p$, and mass $s$. From Eq. (3.1) we have for $l \approx T^{-1 / \lambda_{T}}$ that

$$
P(T, p, L)=T^{-\lambda / \lambda_{T}} P\left(1, T^{-\lambda_{p} / \lambda_{T}} p, T^{1 / \lambda_{T}} L\right) .
$$

Comparing this scaling form with the exactly known value of the exponent $\alpha$, we find that the power of $\mathrm{T}$ in front of this expression is $\lambda / \lambda_{T}=\alpha=1 / 2$. Since the duration of avalanches is measured as a length - the number of rows that the avalanche goes - we have a trivial scaling for $T$, i.e., $\lambda_{T}=-1$. This explains the absence of finite-size effects in the probability distribution of the duration of avalanches in our directed model. Thus the
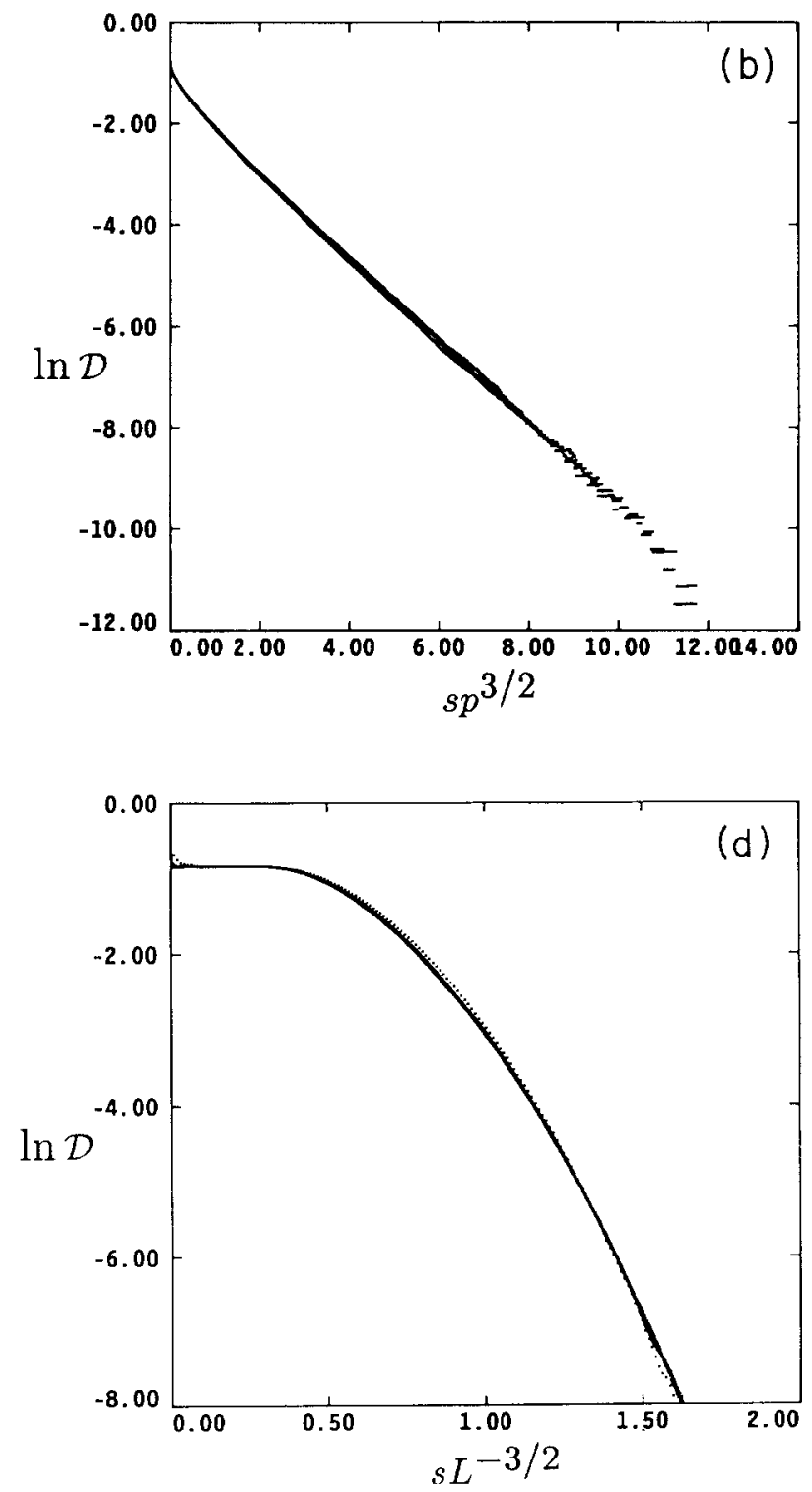

FIG. 4. (a) Logarithm of the scaling function $\mathcal{P}$ defined in Eq. (3.4) plotted against the scaling variable Tp. The four curves are the data from Fig. 1(a), for small concentrations up to $p=0.05$, obtained from simulations of large lattices ( $L=1000)$. (b) Same as (a), but for the scaling function $\mathcal{D}$ defined in Eq. (3.5) and evaluated from the corresponding data in Fig. 1(b). (c) Same as Fig. 1(b), but for pure lattices of different linear size $L=20,50,100,200$, and 500 (from left to right). (d) Logarithm of the scaling function $\mathcal{D}$ evaluated from the data in (c) plotted against the scaling variable $s L^{-3 / 2}$. 
scaling exponent $\lambda$ defined in Eq. (3.1) is $\lambda=-1 / 2$. The remaining scaling variable in expression (3.3) is in fact $T / x(p)$. According to our numeric results in Fig. 2(a), we have $\lambda_{p} \approx 1$. Therefore we may write

$$
P(T, p, L)=T^{-1 / 2} \mathcal{P}(T p, T / L),
$$

where $\mathcal{P}(T p, T / L)$ represents the corresponding scaling function. Applying a similar procedure to expression (3.2) with the choice $l \approx s^{-1 / \lambda}$, one has $D(s, p, L)=s^{-\lambda^{\prime} / \lambda_{*}} D\left(1, s^{-\lambda_{p} / \lambda_{s}} p, s^{1 / \lambda_{s}} L\right)$. Using the fact that $\lambda^{\prime} / \lambda_{s}=\tau=1 / 3$, and that $\lambda_{p} / \lambda_{s}=1 / \mu_{m} \approx 2 / 3$ [cf. Fig. 2(b)], and the above value for the exponent $\lambda_{p}=1$, we finally have $\lambda_{s} \approx-3 / 2$ and $\lambda^{\prime}=-1 / 2$. Thus, the scaling form for the mass distribution $D(s, p, L)$ reads

$$
D(s, p, L)=s^{-1 / 3} \mathcal{D}\left(s p^{3 / 2}, s L^{-3 / 2}\right) .
$$

Here again $\mathcal{D}(x, y)$ is the scaling function of the corresponding scaling variables. We expect that the scaling analysis works for small concentrations of defects, where the characteristic length $x(p)$ is quite large, and thus there is a finite range of values of $T$ and $s$ where the power-law behavior prevails. The scaling function $\mathcal{P}(T p, T / L)$ defined in Eq. (3.4) is determined using the data in Fig. 1(a) (four curves corresponding to the lowest concentrations), and plotted in Fig. 4(a) against the scaling variable $T p$. The curves for different values of $p$ from Fig. 1(a) fall on the same curve. As mentioned in Sec. II, the data for small concentrations in Fig. 1 are obtained for a large lattice size $(L=1000)$, to ensure the absence of finite-size effects. This proves important in the case of mass distribution (see below). In the case of length distribution we checked that there are no nontrivial size effects, i.e., data obtained from different lattice sizes fall on the same curve in Fig. 4(a), in agreement with the above scaling analysis. In a similar way the scaling function $\mathcal{D}\left(s p^{3 / 2}, s L^{-3 / 2}\right)$, defined in Eq. (3.5), is evaluated from the data in Fig. 1(b). (for concentrations up to 0.05) and plotted in Fig. 4(b) against the corresponding scaling variable $s p^{1.5}$. If for a certain concentration of defects the characteristic length $x(p) \geq L$, then the finite size of the lattice will affect the measured probabilities. The characteristic length $x(p)$ diverges at $p \rightarrow 0$, and finitesize effects show up in the mass distribution, as shown in Fig. 4(c). These data are analyzed using the scaling property in Eq. (3.5) with $p=0$, and the corresponding scaling function is plotted in Fig. $4(\mathrm{~d})$ against $s L^{-3 / 2}$.

The dynamic exponent $z$ is usually defined as the exponent which governs the divergence of the characteristic time vs characteristic length in the system near the critical point. It should be stressed, however, that in the directed critical height model the duration of avalanchesusually interpreted as time-is measured in units of length. Therefore, there is no need to define the dynamic exponent in the directed critical height model, or otherwise $z=1$. Instead we define an exponent $D$ which connects the mass of avalanches $s$ with their duration $T$ via the following relation: $\langle s\rangle_{T} \sim T^{D}$. Here the average \langle\rangle$_{T}$ is taken over all events which result in avalanches of a given length $T$. In Fig. $5 T$ is plotted against $\langle s\rangle_{T}$. The data presented as points are for the lattice without de-

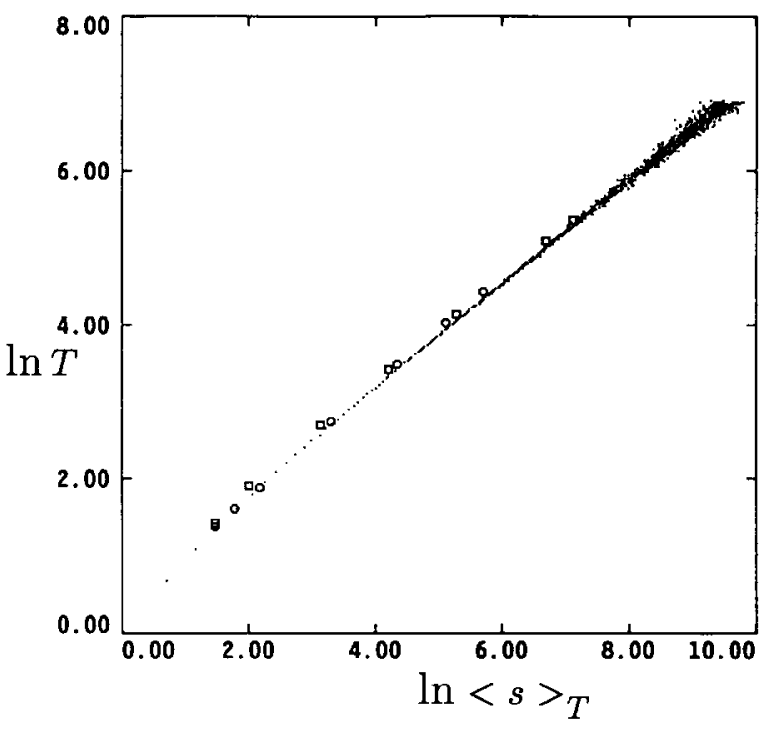

FIG. 5. Double-logarithmic plot of $T$ against logarithm of the cluster size $\langle s\rangle_{T}$ averaged over all avalanches of length $T$ for the directed critical height model on a pure lattice. Also shown are the points (open squares) representing $\ln x$ vs $\ln m$ for various concentrations of annealed defects corresponding to Fig. 2. The open circles represent data for $\ln x$ vs $\ln m$ obtained in the case of quenched defects for concentrations (from left to right) $p=0.295,0.25,0.2,0.1,0.05,0.03$, and 0.02 .

fects and with linear size $L=1000$. In the case of finite concentration of defect sites the self-similarity is expected to hold on scales of order of the characteristic length $x(p)$ and the characteristic mass $m(p)$. The open squares in Fig. 5 represent the plot of $\ln (x)$ vs $\ln (m)$ for different concentrations $p$ taken from Fig. 2, corresponding to the annealed disorder. The open circles represent the same kind of data obtained in the case of quenched disorder from systems of linear size $L=192$ with concentrations $p=0.295,0.25,0.2,0.1,0.05,0.03$, and 0.02 .

The slope in Fig. 5 determines the dimension of the avalanches of linear size $T$. (The fractal dimension of avalanches is usually related to the dynamic exponent in undirected sandpile models $[4,5]$.) Our estimate of the dimension according to Fig. 5 is $D=1.51 \pm 0.02$. In the case of a finite concentration of defects this result applies only for avalanches of linear size smaller than the characteristic length $x(p)$.

The total mass of the system (averaged over 1000 avalanches for better resolution and normalized by the total number of lattice points) is presented in Fig. 6(a) against the simulation time (measured in number of events) for finite concentration of defects $p=0.05$. For comparison similar data for the perfect lattice $(p=0)$ are presented in Fig. 6(b). The Fourier spectra of the data from Figs. 6(a) and 6(b) are shown in a doublelogarithmic scale in Fig. 6(c), with further average over 10 points. The upper curve corresponds to the pure case, showing roughly $1 / f$ noise. For the diluted case (the 

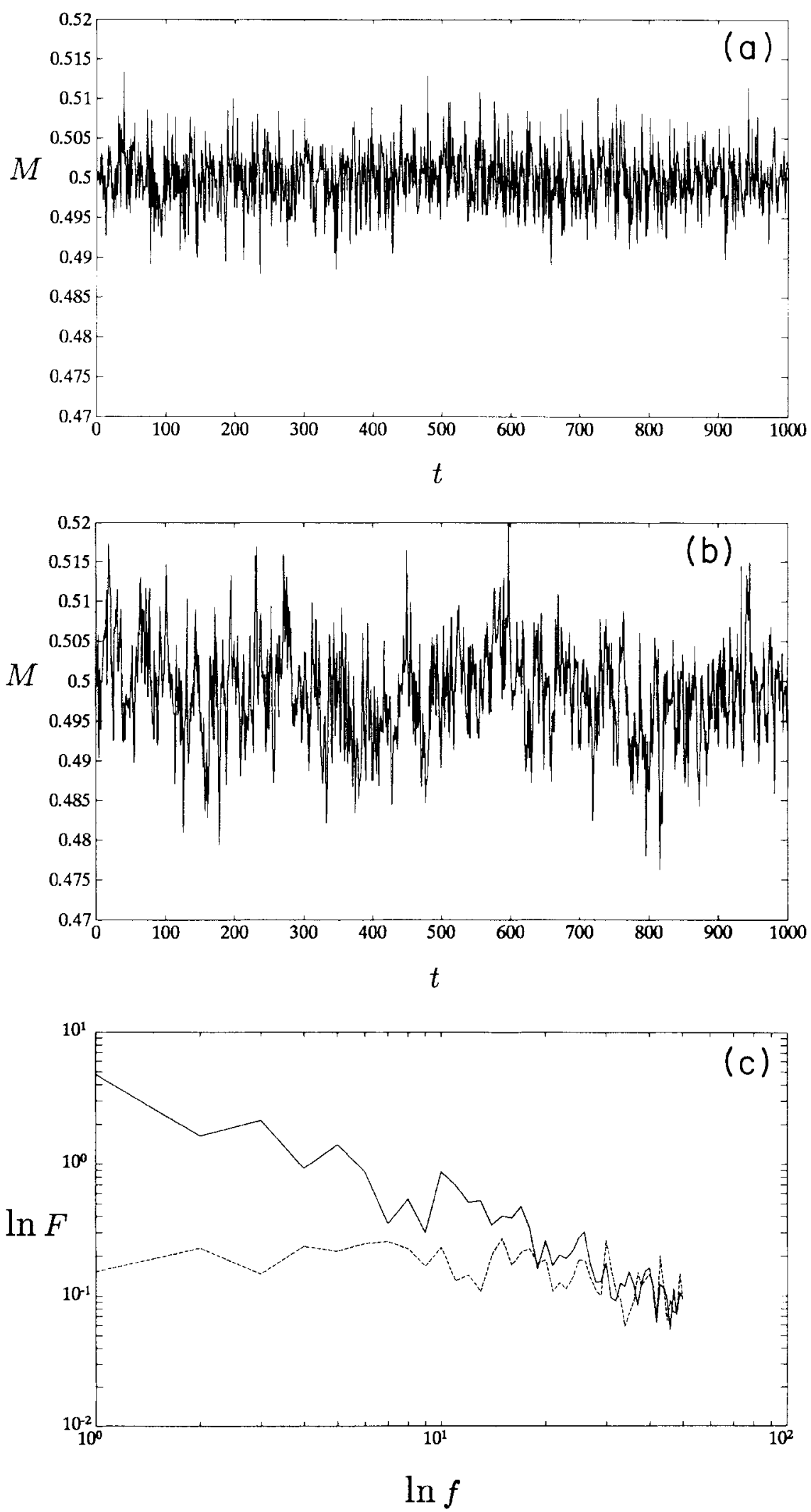

FIG. 6. Total mass of the system defined as $M=\sum_{i, j} h(i, j)$ (contribution of defect sites counted as zero) normalized by the total number of lattice sites and averaged over 1000 events, plotted against time (measured in number of events) for (a) a lattice with $5 \%$ defect sites, and (b) a lattice without defects. (c) Fourier spectrum of the mass data from (a) and (b), lower and upper curves, respectively, in a double-logarithmic plot. $F$ is defined as $F=|M(f)|^{2}$, where $f$ is the frequency. 
lower curve) the flat distribution of noise gives evidence for the loss of the self-organized criticality at long times.

\section{RANDOM CRITICAL HEIGHTS MODEL}

In Secs. II and III we have shown that the presence of defects acting as sinks of particles in the directed sandpile automaton is the reason for the loss of the self-organized critical behavior at large scales. These effects are the same for both a "quenched" or an "annealed" distribution of defects. In both cases the presence of random defects violates the conservation law of the microscopic dynamic rule-which governs the details of the height transport-and the symmetry with respect to the translation of heights. Both the conservation law and the symmetry are important properties of the models of selforganized criticality. In the present section we want to address the question whether a violation of the conservation law or a violation of the symmetry is responsible for a lack of self-organized criticality. We consider a type of randomness which modifies the critical heights in the system, but does not assume loss of particles. There are obviously a number of different ways to implement the random critical heights in the directed sandpile automaton in two dimensions.

We first describe a model with extended defects, which we refer as model I, and which consists of the following set of rules. Starting from the ideal lattice with the critical height $h_{c}(i, j)=2$ at all sites, defect sites with $h_{c}=0$ are randomly distributed over the lattice with concentration p. Then the critical heights at sites close to the defects are modified in the following way. At two sites $\left(i-1, j_{ \pm}\right)$, neighbors to the defect site $(i, j)$, the critical height is lowered by one $h_{c}\left(i-1, j_{ \pm}\right) \rightarrow h_{c}\left(i-1, j_{ \pm}\right)-1$. In this way some lattice sites will result in the critical height one, and-if a cluster of defects occurs - the critical height can also be zero at some lattice points. The sites with zero critical height are located and, as well as defect sites, are considered to be inaccessible for the particles. Thus the sites with $h_{c}=1$ have only one forward neighbor with the critical height 1 or 2 , and the sites with $h_{c}=2$ have two neighbors where the particles can be sent. According to these rules, by high enough values of concentration of defect sites, a kind of extended defect can be formed with the surrounding sites having the critical height 1 .

This model is merely considered here as an automaton with a set of microscopic rules that are complementary to the model of random sinks introduced in Sec. II. After having added a particle at the top row, we apply the updating rules (2.1) and (2.2), with the condition that sometimes there is only one forward neighbor, as discussed above. As a consequence of these dynamic rules some of the sites with $h_{c}=1$ may remain critical after one toppling has been accomplished. To stabilize the system, disconnected avalanches may start from such sites. In this way, counting of the events which contribute to the distribution of length of avalanches $P(T)$ has to be done with care. Following the avalanche which starts from the first row, we do not allow contributions of disconnected avalanches to the probability distributions. The resulting distribution $P(T)$ is shown in Fig. 7(a) for different values of concentrations of defects. For $p=0$ the selforganized criticality is reproduced, as expected. For any finite value of $p$ the self-organized critical behavior is lost in analogy to the model of random holes, introduced in Sec. II (cf. Fig. 1). The reason for the loss of criticality in the present case can be found in the fact that under the dynamic rules (2.1) and (2.2) there is the possibility of accumulation of sand particles at sites with the critical height 1 . The average number of particles in these sites is growing forever; a steady state is never reached. Clearly, this behavior is similar to the model with random holes, where particles are falling out of the system, so similar behavior could be expected.

In the models considered so far both the height conservation and the translational invariance are violated resulting in a loss of self-organized criticality at large scales. It is therefore an interesting question whether a violation of the translational invariance alone leads to a loss of self-organized criticality. To shed some light on this particular problem we finally study the following model (model II). A random quenched distribution of critical heights $h_{c}(i, j)=10$ with concentration $p$ is introduced while the remaining sites have the critical height $h_{c}=2$. The toppling rules defined earlier by Eqs. (2.2) are then modified to

$$
\begin{aligned}
& h(i, j) \rightarrow h(i, j)-h_{c}(i, j), \\
& h\left(i+1, j_{ \pm}\right) \rightarrow h\left(i+1, j_{ \pm}\right)+h_{c}(i, j) / 2,
\end{aligned}
$$

and applied at each site as many times as necessary to render the site stable, i.e., until the condition $h(i, j)<$ $h_{c}(i, j)$ is fulfilled. As an example consider the site $(k, n)$ which has the critical height $h_{c}(k, n)=10$ and two forward neighbors with the critical height $h_{c}\left(k+1, n_{ \pm}\right)=2$. By one toppling at the site $(k, n)$ ten particles proceed to the next row, five to the left, and five to the right neighboring site $\left(k+1, n_{ \pm}\right)$, which makes them critical. Since $h_{c}=2$ at these sites, they will remain critical after one toppling under the rules (4.1) and (2.2), under which in total four particles proceed and six particles remain at sites $\left(k+1, n_{ \pm}\right)$. We apply again the same toppling rules until the condition $h\left(k+1, n_{ \pm}\right)<h_{c}\left(k+1, n_{ \pm}\right)=$ 2 is fulfilled. The resulting distribution of length of avalanches $P(T)$, exhibited in Fig. $7(\mathrm{~b})$, shows that the self-organized criticality survives in this model for all concentrations of defect sites. Fitting the data to a power law yields the exponents $0.4950,0.4976,0.5045,0.4959$, and 0.5016 (from above). The standard deviations of these values are less than 0.001 . The first exponent corresponds to the pure case and its deviation from the exact value 0.5 has been discussed earlier. The fluctuations observed in the case of random critical heights we expect to be due to the finite size of the random lattice. There is no indication that our model II belongs to another universality class than the pure system, although a very small change in the exponent cannot be ruled out completely.

Applying in a similar way the dynamic rules (4.1) repeatedly until $h(i, j)<h_{c}(i, j)$ at sites with the critical height $h_{c}=1$ in the case of model 1 , we find that for relatively small concentrations of defect sites the distributions of avalanches continue to show self-organized 
critical behavior with the exponents of the pure model. Due to the correlation of the extended defects, the effective concentration of defect sites $p_{\text {eff }}$ is larger than $p$. For concentrations for which $p_{\text {eff }}$ is larger than a critical value $p_{c}$, so that the global connectivity of the accessible sites in the lattice is lost, the probability of large avalanches falls off faster than a power law, resulting in a loss of the SOC. This can be seen in Fig. 7(c). The power-law distribution is preserved for a lower concentration of defects (upper curve, $p=0.2<p_{c}$ ). For higher concentrations (lower curve, $p=0.26>p_{c}$ ) there is a breakdown of the probability distribution on larger length scales.

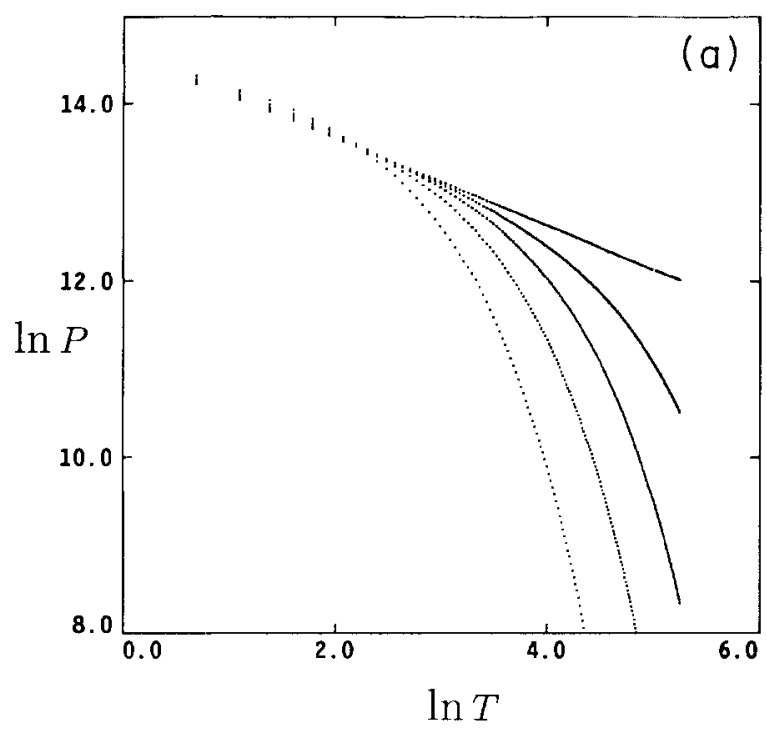

\section{DISCUSSION AND CONCLUSIONS}

We used numeric simulations to study the effects of disorder on the self-organized criticality in two-dimensional sandpile automata with preferred direction. We have introduced two sandpile automata with random defects, which we believe may describe different aspects of the appearance of the power law in the avalanche distributions. The microscopic dynamic rules that describe how an avalanche is generated are modified at the defect sites in two different ways. (1) In the model with holesdefined in Sec. II- sand grains leave the system when an

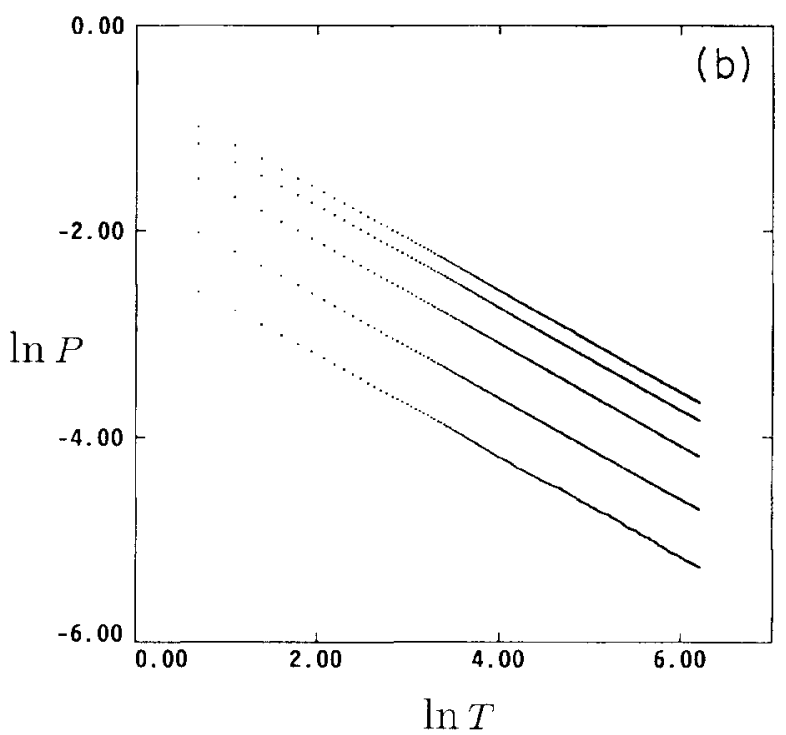

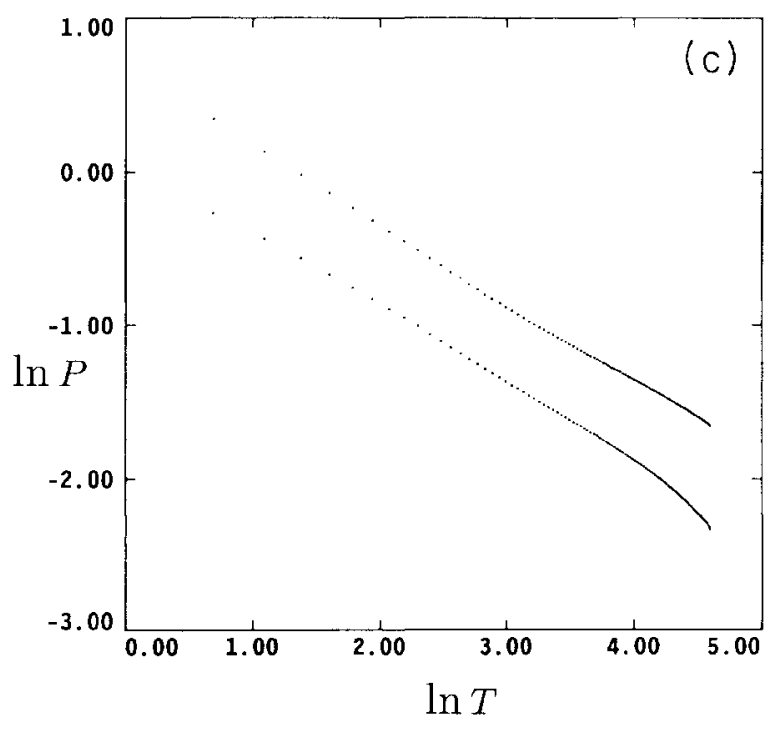

FIG. 7. Double-logarithmic plot of the probability distribution of length $P$ vs the length of avalanches $T$ in the case of random critical heights for the following. (a) Model I - extended defects - concentrations (from top to bottom) $p=0,0.05$, $0.1,0.2$, and 0.295 . (b) Model II - randomly distributed critical heights $h_{c}=10$ in the host of $h_{c}=2$ and repeatedly applied toppling rules. Concentrations $p=0,0.2,0.5,0.8$, and 1 . (c) Extended defects (model I) with the repeatedly applied dynamic rules. Concentrations $p=0.2,0.26$. The upper curve is shifted by a factor of 2 . The models are described in Sec. IV. 
avalanche hits a defect site. (2) In the model with random critical heights the condition for toppling is locally modified at defect sites-models I and II in Sec. IV represent two of many possible ways that defects may affect local critical heights. Our results suggest that the selforganized critical state is lost in the presence of defects if the modified dynamic rules violate locally the height conservation. This is the case in our model of random holes and model I of random critical heights. The systems described by this type of defect are driven out of the critical point, with the concentration of defects $p$ playing the role of a critical parameter. On the other hand, lacking the translational symmetry but with locally conserving dynamics, as our model II of random critical heights, leaves the system in the critical state with no hint for a change of the exponents compared to the pure model. It should be noted that in the latter case the conservation law is observed locally, in contrast to the case considered in Ref. [12], where the conservation law is locally violated but globally reassured, leading to mean-fieldlike exponents. It is not clear to us why the continuum model studied by the dynamic renormalization group in Ref. [14] belongs to the same universality class.

In the case of extended defects, as discussed at the end of Sec. IV, the power-law behavior disappears at finite concentration of defects, due to a loss of the lattice connectivity.

In the case of random holes we analyzed in detail the disappearance of the power-law behavior by varying the concentration of defects $p$. At small but finite concentra- tions we find that a characteristic length $x(p)$ and a characteristic mass $m(p)$ exist, such that for $T \ll x(p)$ and $s \ll m(p)$ the self-similarity persists. The self-organized criticality at the reduced scale is in the same universality class as the system without defects. The occurrence of power-law behavior at scales smaller than the characteristic ones is expressed in the general scaling forms (3.4) and (3.5). We have determined the scaling functions $\mathcal{P}$ and $\mathcal{D}$ defined in Eqs. (3.4) and (3.5), which express the scaling properties of the corresponding probability distributions $P(T, p, L)$ and $D(s, p, L)$, respectively, and we have determined the corresponding scaling exponents. In the limit $p \rightarrow 0$ the characteristic length $x(p)$ and the characteristic mass $m(p)$ of the avalanche diverge as a power of $p$, and the criticality due to self-organization is regained at $p=0$. Our simulations in the sandpile automaton with random holes indicate that there is no potentially important difference between the two types of defects usually termed as "annealed" and "quenched" disorder.

\section{ACKNOWLEDGMENTS}

Two of us (B.T. and S.P.) acknowledge the hospitality of Jawaharlal Nehru University, where this work was initiated. Two of us (B.T. and U.N.) have benefited from discussions with D. Dhar. We would also like to thank S. S. Manna for helpful discussions. This work was supported in part by the Deutsche Forschungsgemeinschaft through Sonderforschungsbereich 166.
- On leave from Jožef Stefan Institute, YU-61111 Ljubljana, Yugoslavia. Present address: Institute of Physics, P.O. Box 57, YU-11001 Belgrade, Yugoslavia

1 Electronic address: uli@hal9000.uni-duisburg.de.

[1] P. Bak, C. Tang, and K. Wiesenfeld, Phys. Rev. Lett. 59, 381 (1987).

[2] M. Kardar, in Disorder and Fracture, edited by J.C. Charmet et al. (Plenum, New York, 1990), p. 3.

[3] P. Bak, C. Tang, and K. Wiesenfeld, Phys. Rev. A 38, 364 (1988).

[4] C. Tang and P. Bak, Phys. Rev. Lett. 60, 2347 (1988).

[5] T. Hwa and M. Kardar, Phys. Rev. Lett. 62, 1813 (1989); Physica D 38, 198 (1989).

[6] G. Grinstein, D.-H. Lee, and S. Sachdev, Phys. Rev. Lett. 64, 1297 (1990).

[7] D. Dhar and S.N. Majumdar, J. Phys. A 23, 4333 (1990).

[8] S.S. Manna, Physica A (to be published).
[9] L.P. Kadanoff, S.R. Nagel, L. Wu, and S.-m. Zhou, Phys. Rev. A 39, 6524 (1989).

[10] D. Dhar and R. Ramaswamy, Phys. Rev. Lett. 63, 1659 (1989).

[11] S.-K. Ma, Modern Theory of Critical Phenomena (Benjamin-Cummings, Reading, MA, 1976).

[12] S.S. Manna, L.B. Kiss, and J. Kertész, J. Stat. Phys. 61, $923(1990)$.

[13] In P. Bak, K. Chen, and M. Creutz, Nature 342, 780 (1989) the authors claim that the "game of life"an automaton without a conservation law-shows selforganized criticality. See also C. Bennett and M.S. Bourzutschky, Nature 350, 468 (1991), who contradict this claim.

[14] J. Toner, Phys. Rev. Lett. 66, 679 (1991).

[15] J. W. Essam, A. J. Guttmann, and K. de'Bell, J. Phys. A 21, 3815 (1988). 